\title{
Noradrenaline arouses astrocytes
}

Astrocytes have diverse functions in the brain, but little is known about how the activity of astrocyte networks is regulated. A new study suggests that noradrenaline released during arousal controls the ability of astrocytes to respond to local neuronal activity.

Activation of neurotransmitter receptors expressed on astrocytes can induce an increase in intracellular $\mathrm{Ca}^{2+}$ levels, and such $\mathrm{Ca}^{2+}$ transients are used as a marker of astrocyte activity. To investigate this regulation of astrocyte activation in more detail, Paukert et al. generated mice that expressed the $\mathrm{Ca}^{2+}$ indicator GCaMP3 in astrocytes in the cerebral cortex and in Bergmann glia in the cerebellar cortex. Combined with two-photon imaging, this enabled the authors to visualize $\mathrm{Ca}^{2+}$ transients through a cranial window in these mice in vivo without anaesthesia.

Using this method, the authors showed that both voluntary and forced locomotion on a treadmill was associated with $\mathrm{Ca}^{2+}$ transients in Bergmann glia, in agreement with previous findings. However, not all locomotion bouts elicited a $\mathrm{Ca}^{2+}$ response and $\mathrm{Ca}^{2+}$ transients also occurred in the absence of locomotion. Electromyography recordings revealed that such apparently spontaneous transients in fact coincided with muscle activity. The authors hypothesized that the muscle activity might reflect startle behaviour and, therefore, that Bergmann glia activity might be associated with arousal.
Arousal involves activation of the locus coeruleus, resulting in widespread noradrenaline release. Depletion of noradrenaline (through intraperitoneal injections of the neurotoxin DSP4) prevented locomotion-induced $\mathrm{Ca}^{2+}$ transients, and local application of an a1-adrenergic receptor antagonist nearly abolished them. This suggested that Bergmann glia activity is indeed induced by noradrenaline release and thus, probably, by arousal.

Noradrenergic locus coeruleus neurons project throughout the brain. The authors therefore assessed whether arousal-induced noradrenaline release also activates astrocytes in the primary visual cortex (V1), which is not directly associated with motor control. Forced locomotion in complete darkness (to avoid any effects of light-activated V1 neurons on astrocytes) elicited $\mathrm{Ca}^{2+}$ transients in $\mathrm{V} 1$ astrocytes that were, like in Bergmann glia, blocked by application of DSP4 or administration of an a1-adrenergic receptor antagonist.

The authors next examined whether noradrenaline altered the ability of astrocytes to respond to local circuit activity by comparing the astrocyte response to forced locomotion in the dark (to elicit noradrenaline release) with that of visual stimulation (to elicit local circuit activity). As before, forced locomotion often elicited astrocyte activation, but visual stimulation did so only occasionally — when observed, it was always coincident with muscle activity and thus, potentially, resulting from enhanced arousal. Remarkably, combining locomotion with visual stimulation greatly enhanced $\mathrm{Ca}^{2+}$ transients compared with locomotion alone, and this enhancement was due to an increase in both the response magnitude and the proportion of responding astrocytes. This suggests that the arousal induced by locomotion increased the ability of $\mathrm{V} 1$ astrocytes to respond to V1 neuron activation triggered by visual stimulation.

Together, these findings suggest that arousal - via noradrenaline - increases the gain of astrocyte networks, which in turn enables them to respond to local neuronal activity.

Leonie Welberg
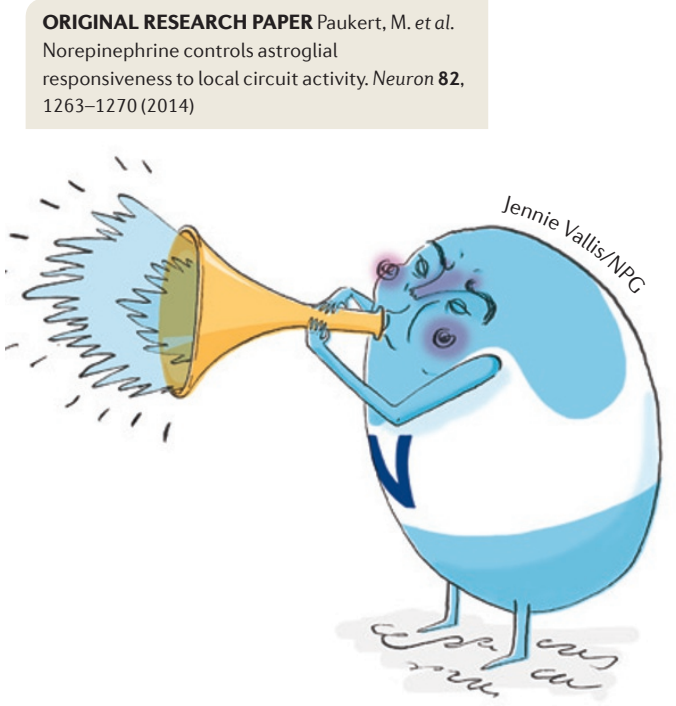

combining

locomotion

with visual

stimulation

greatly enhanced $\mathrm{Ca}^{2+}$

transients

compared with

locomotion

alone

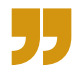

